\title{
Decreased expression of LKBI predicts poor prognosis in pancreatic neuroendocrine tumor patients undergoing curative resection
}

\author{
Dezhi Li ${ }^{1, *}$ \\ Yu Zhou ${ }^{1, *}$ \\ Yanhui Liu',* \\ Ye Lin' \\ Min Yu' \\ Xin Lu' \\ Bowen Huang' \\ Zhonghai Sun' \\ Zhixiang Jian' \\ Baohua Hou' \\ 'Department of General Surgery, \\ Guangdong General Hospital, \\ Guangdong Academy of Medical \\ Sciences, Guangzhou, Guangdong, \\ People's Republic of China; \\ ${ }^{2}$ Department of Pathology, \\ Guangdong General Hospital, \\ Guangdong Academy of Medical \\ Sciences, Guangzhou, Guangdong, \\ People's Republic of China \\ *These authors contributed equally \\ to this work
}

Correspondence: Baohua Hou;

Zhixiang Jian

Department of General Surgery, Guangdong General Hospital, Guangdong Academy of Medical Sciences, Nol06,

Zhong Shan Er Lu, Guangzhou 510080 ,

Guangdong, People's Republic of China Tel/fax +86 $20838278 / 2$

Email houbaohua_ggh@I63.com;

jianzx_II8@I63.com
This article was published in the following Dove Press journal:

OncoTargets and Therapy

Background: Liver kinase B1 (LKB1) is a key regulatory protein of cellular metabolism, proliferation, and polarity. The present study aimed to characterize the expression pattern of LKB1 in pancreatic neuroendocrine tumors (pNETs) and evaluate the relationship between LKB1 expression and prognosis in pNETs.

Patients and methods: We retrospectively analyzed the pathologic and clinical data of 71 pNET patients who underwent curative surgical resection in Guangdong General Hospital. LKB1 mRNA and protein levels in tumor tissues and paired nontumor tissues were evaluated in 24 patients by quantitative real-time reverse-transcription polymerase chain reaction and Western blot, respectively. Immunohistochemical expression of LKB1 in tumor tissues was detected in all of the 71 patients, and the immunohistochemical expression level was re-coded in two classes (high versus low/negative) and correlated with clinicopathological parameters and survival outcomes. The association between LKB1 expression and clinicopathological characters was evaluated by chi-square test and Student's $t$-test. Kaplan-Meier curves and log-rank test were used to analyze the survival outcomes, including overall survival (OS) and disease-free survival (DFS).

Results: Compared to adjacent normal tissues, LKB1 mRNA level and protein expression level in tumor tissues were both increased. The immunostaining of LKB1 was mainly found within the cytoplasm. Overall, 52 of 71 (73.2\%) cases were positive for LKB1 protein, which showed either a diffuse staining pattern or a partial staining pattern. Decreased LKB1 expression was correlated with older age $(P=0.042)$, increased Ki-67 index $(P=0.004)$, increased mitotic count $(P=0.001)$, and advanced histologic grade $(P=0.001)$. Moreover, patients with low/negative LKB1 expression had shorter OS and DFS than those with high expression.

Conclusion: Our results suggested that LKB1 expression could be a useful prognostic marker for recurrence and survival in pNET patients who had received curative resection.

Keywords: pancreatic neuroendocrine tumors, liver kinase B1, LKB1, prognosis

\section{Introduction}

Pancreatic neuroendocrine tumors (pNETs) are the second most common pancreatic neoplasms behind ductal adenocarcinoma and account for about $1 \%-4 \%$ of all pancreatic neoplasms. ${ }^{1,2}$ Although pNETs are rare neoplasms, their incidence rate has increased rapidly in recent years to about $1 / 100,000$ yearly. The reason for a rapidly increasing incidence rate of PNETs is not very clear. The most likely explanation is the development of $\mathrm{CT}$ and other imaging techniques, as a result, the early diagnosis rate increased rapidly, so the incidence is increasing. ${ }^{3,4}$ Management of pNETs is challenging because of their heterogeneous pathologic features and unpredictable clinical behaviors..$^{5}$ In pNET patients with curative resection, the prognosis is usually favorable but 
long-term outcomes after surgery vary widely, ${ }^{6-9}$ largely depending on the biological characteristics of the tumor. ${ }^{10}$ Knowledge about the prognosis of a pNET patient provides support for the optimal management of pNETs. Therefore, it is critical to discover novel factors that are associated with development, progression, and prognosis of pNETs.

Liver kinase B1 (LKB1), also known as STK11, is widely expressed in human tissues. ${ }^{11,12}$ LKB1 is a highly conserved serine/threonine kinase that directly phosphorylates a threonine residue at the activation loop of 14 kinases in the AMPK (AMP-activated kinase) family, ${ }^{13,14}$ many of which are coupled to cell metabolism, proliferation, and polarity. ${ }^{15-18}$ Inactivation of LKB1 is responsible for the Peutz-Jeghers syndrome, which exhibits a predisposition to both benign and malignant tumors in multiple organ systems. ${ }^{11,12}$ Besides, LKB1 has been classified as a tumor suppressor through regulation of AMPK signaling in a variety of malignancies including gastric cancer, pancreatic adenocarcinoma, breast cancer, prostate cancer, ovarian cancer, cervical cancer, and hepatocellular carcinoma. ${ }^{19-26}$ A previous study had also described an obvious downregulation of LKB1 in neuroendocrine tumors of the lung. ${ }^{27}$ However, the expression and role of LKB1 in pNETs still remain unknown. Here, in the present study, we aimed to assess the possible role of LKB1 as a factor of prognosis in pNET patients.

\section{Patients and methods}

\section{Patients and samples}

Our study included 71 patients who underwent curative surgical treatment for pNETs between January 2000 and July 2016 at the Department of General Surgery, Guangdong General Hospital. None of them had ever received any adjuvant therapy before surgery. This study was approved by the Institutional Review Board of Guangdong General Hospital, and written informed consents were obtained from all patients. The tumor tissues were obtained during surgery. Histological types and grades of all samples were determined by experienced pathologists. The histology and clinical stages were classified based on the seventh edition of the American Joint Committee on Cancer staging system. Histological grades of the tumors were classified according to the World Health Organization 2010 classification system. The inclusion criteria for patients were as follows: 1) localized pNET lesions with radical resection; 2) pathological confirmation of PNETs by postoperative histopathology diagnosis; 3) no adjuvant therapy prior to surgery; 4) tumor does not involve the celiac axis or the superior mesenteric artery or exhibit distal metastasis; and 5) without history of other malignancies. Patients who died during the perioperative period were excluded. The follow-up information was obtained through interview or telephone call.

\section{Immunohistochemistry (IHC)}

IHC was used to examine the expression of LKB1 at the protein level in formalin-fixed paraffin-embedded primary specimens. Slides ( $4 \mu \mathrm{m}$ thick, two serial sections for each sample) were dewaxed and rehydrated with xylene and graded alcohol, respectively. The activity of endogenous peroxidase was quenched by 10 -minute incubation in a mixture of $3 \%$ hydrogen peroxide at room temperature. Nonspecific binding was blocked by incubation in 3\% bovine serum albumin in phosphate-buffered saline (PBS). Slides were then incubated with LKB1 antibody (monoclonal mouse antibody, 1:100 dilution; Abcam, Cambridge, UK). Following additional wash with PBS, secondary antibody was added to the sections.

LKB1 immunostaining results were examined microscopically by two pathologists independently. Expression level of LKB1 was classified according to the intensity of staining and the percentage of positively stained cells through evaluating 10 randomly selected high-power fields. Sections with strong immunostaining in $>50 \%$ of cells were drawn in high-expression group; otherwise, sections with strong immunostaining in $\leq 50 \%$ of the cells and/or weak staining were drawn in low-expression group. Disagreements between pathologists regarding immunohistochemical staining were resolved through discussion.

\section{Quantitative real-time reverse- transcription polymerase chain reaction ( $q R T-P C R$ )}

qRT-PCR was used to examine the expression of LKB1 at the RNA level in frozen tumor tissues and paired adjacent normal specimens from a total of 24 consecutive patients receiving surgical treatment between June 2014 and July 2016. Total RNA was extracted from frozen tissues using TRIzol reagent (Thermo Fisher Scientific, Waltham, MA, USA). qRT-PCR was performed for LKB1 with GAPDH as an internal control. After RNA intensity and purity were quantified, RNA was then converted to cDNA from $1 \mu \mathrm{g}$ RNA using PrimeScript reagent kit (Takara, Tokyo, Japan). The PCR amplification was performed by using a SYBR Premix Ex Taq II kit (Takara) and analyzed using a Roche LightCycler (Hoffman-La Roche Ltd., Basel, Switzerland). Primer sequences used for LKB1 and GAPDH were as follows: LKB1 forward: 5'-GAGCTGATGTCGGTGGGTATG-3', LKB1 reverse: 5'-CACCTTGCCGTAAGAGCCT-3'; GAPDH forward: 5'-GTCGGAGGCAACATCACC-3', GAPDH reverse: 5'-GTCCAAATGCGGGAACAG-3'. 
The relative RNA expression in samples was determined using the comparative delta-delta CT method $(2-\Delta \Delta \mathrm{Ct})$ and the fold change in gene expression of tissues was calculated using the standard $\Delta \Delta \mathrm{Ct}$ method.

\section{Western blot}

The level of LKB1 protein was determined by Western blot analysis in paired tumor and adjacent normal specimens. The total protein was extracted from frozen specimens using a protein extraction kit (Hoffman-La Roche Ltd.) and quantified using a bicinchoninic acid assay according to standard protocols. Equivalent amounts of proteins were separated by SDS-PAGE and transferred to polyvinylidene fluoride membranes for immunoblotting. The membranes were blocked in $5 \%$ fat-free milk and then incubated with the primary antibodies overnight at $4{ }^{\circ} \mathrm{C}$. LKB1 antibody (monoclonal mouse antibody, 1:500; Abcam) and GAPDH antibody (monoclonal mouse antibody, 1:1,000; Abcam) were used at the indicated dilutions. After incubation with a horseradish peroxidase-conjugated secondary antibody, signals of horseradish peroxidase were detected by enhanced chemiluminescence detection reagents.

\section{Statistical analysis}

All analyses were performed with Statistical Product and Service Solutions software package 17.0 (SPSS Inc., Chicago, IL, USA). Frequency variables were compared by chi-square test, and continuous variables were compared using the Student's $t$-test. Survival outcome was analyzed in terms of overall survival (OS) and disease-free survival (DFS). Survival curves were compared using the Kaplan-Meier method and tested by the log-rank test. A $P$-value of less than 0.05 in a two-tailed test was considered statistically significant.

\section{Results}

\section{LKBI mRNA and protein expression in pNET tissues and corresponding adjacent normal tissues}

We examined LKB1 mRNA and protein expression in 24 frozen pNET tissues versus surrounding normal tissues obtained from the same patients by qRT-PCR and Western blot, respectively. As shown in Figure 1A, the expression level of LKB1 mRNA in pNET tissues was decreased compared with that in corresponding normal tissues and the difference was significant $(P<0.001)$. To confirm LKB1 expression at protein level, LKB1 protein expression was measured by Western blotting in the same samples, and results confirmed that LKB1 proteins were indeed downregulated in cancer tissues (Figure 1B).
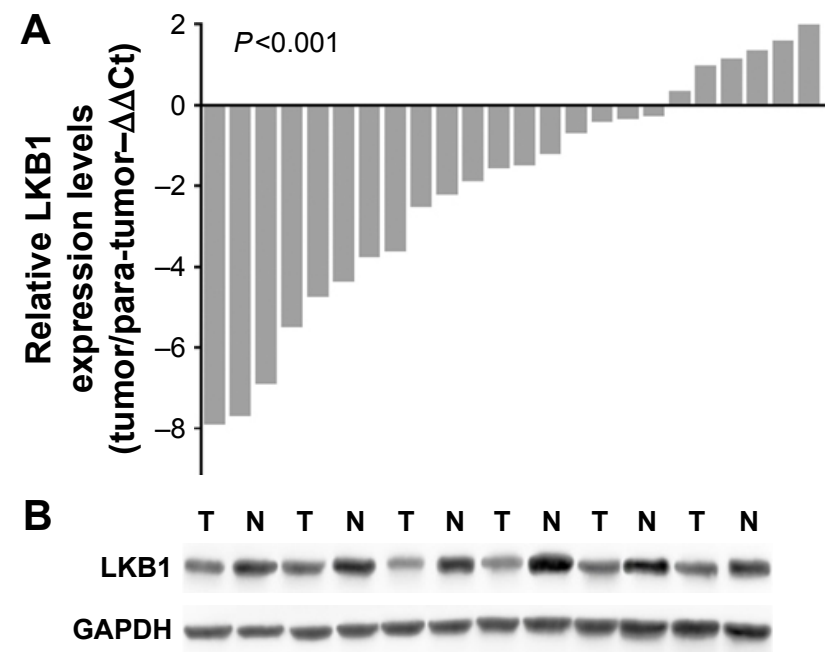

Figure I LKBI mRNA and protein levels in PNET tissues and paired normal tissues.

Notes: (A) The mRNA levels of LKBI were examined via qRT-PCR in 24 pairs of PNET tissues and corresponding nontumorous tissues. GAPDH was used as an internal control. Relative gene expression was determined using the comparative delta-delta CT method, and data are presented as $\Delta \Delta C t(P<0.00 I$, paired Student's $t$-test). (B) Representative Western blot images of LKBI protein level in pairs of PNET tissues $(T)$ and corresponding nontumorous tissues $(N)$.

Abbreviations: LKBI, liver kinase $\mathrm{BI}$; PNET, pancreatic neuroendocrine tumor; qRT-PCR, quantitative real-time reverse-transcription polymerase chain reaction.

\section{LKBI immunohistochemistry}

The LKB1 protein expressions of all pNET tissues were assayed by IHC method. The immunostaining of LKB1 was mainly found in the cytoplasm. The LKB1-positive pNET tissues displayed either a diffuse staining pattern or a partial staining pattern. Overall, 52 of $71(73.2 \%)$ cases were positive for LKB1 protein but at a different degree, and 19 of $71(26.8 \%)$ cases had a totally negative expression. As shown in Figure 2, to obtain an exact result of LKB1 expression level, 71 cases of pNET patients were divided into two groups based upon the percentage of immunostaining and the intensity of immunostaining. Tissues with strong LKB1 staining in more than $50 \%$ tumor area were assigned to the high-expression group $(\mathrm{n}=33)$; tissues with strong LKB1 staining in less than $50 \%$ tumor area, tissues with weak LKB1 staining, and those with totally negative staining were assigned to the low-expression group $(n=38)$.

\section{Correlation between LKBI expression and clinicopathological characteristics}

As shown in Table 1, the association between LKB1 expression and clinicopathological characteristics, including age, gender, tumor size, tumor location, functionality, margin status, differentiation, Ki-67 index, mitoses count, and histologic grade, was evaluated. The results showed that high LKB1 expression was correlated with older age $(P=0.042)$, 

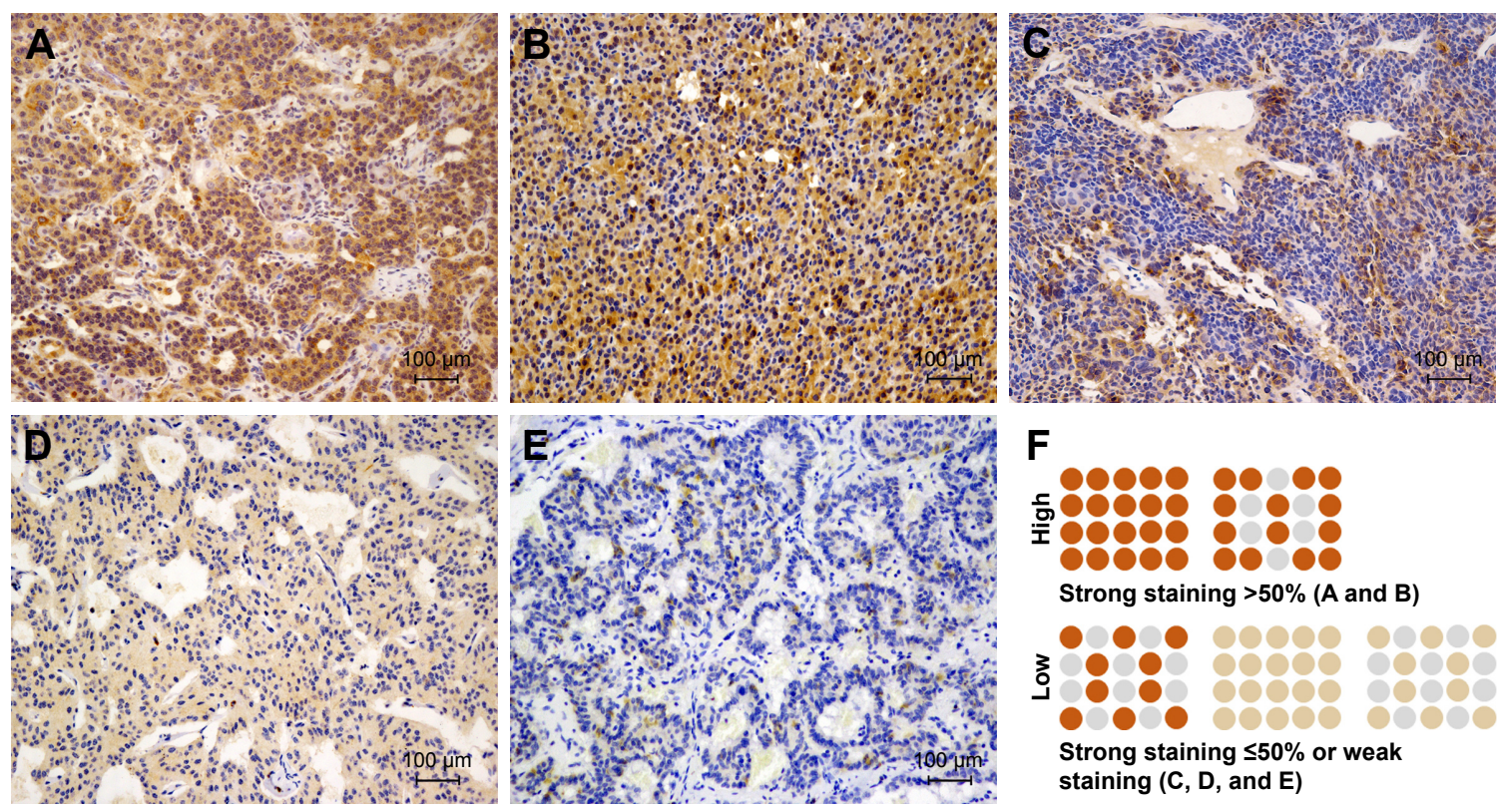

Figure 2 Immunohistochemical staining results of LKBI expression in PNET tissue samples.

Notes: (A-E) Representative images of LKBI expression in PNET tissues. (A) Tissue with diffused strong LKBI staining; (B) tissue with strong LKBI staining in more than $50 \%$ tumor area; (C) tissue with strong LKBI staining in less than $50 \%$ tumor area; (D) tissue with disused weak LKBI staining; (E) tissue with weak LKBI staining in less than $50 \%$ tumor area; and (F) a sketch illuminating the classification method of LKBI immunostaining in pNET tissues. Magnification for these images is I00X.

Abbreviations: LKBI, liver kinase BI; PNET, pancreatic neuroendocrine tumor.

higher Ki-67 index $(P=0.004)$, increased mitoses count $(P=0.001)$, and advanced histologic grade $(P=0.001)$.

\section{Association between LKBI expression and prognosis of pNET patients}

To further evaluate the prognostic value of LKB1 in pNET patients, the Kaplan-Meier analysis and log-rank test were performed. Results showed that patients with low LKB1 expression had a significantly decreased OS and DFS than those with high LKB1 expression $(P=0.0021, P=0.009$, respectively, log-rank test; Figure $3 \mathrm{~A}$ and $3 \mathrm{~B})$. We further determined the correlation between LKB1 expression and prognosis in patients with non-functional pNETs (NF-pNETs). Kaplan-Meier curve showed that OS was shorter in NF-pNET patients with lower LKB1 expression, but the difference was not significant $(P=0.121, \log$-rank test; Figure 3C); meanwhile, the DFS rate of patients with high LKB1 expression was significantly higher than that of patients with low LKB1 expression $(P=0.036$, log-rank test; Figure 3D).

\section{Discussion}

LKB1 has emerged as a critical tumor suppressor in many cancer types. ${ }^{28}$ The downstream signaling of LKB1, mainly including AMPK (adenosine monophosphate activated protein kinase), MARK (microtubule affinity regulating kinase), SAD (synapses of the amphid defective, also known as brain selective kinase [BRSK]), and SIKs (salt-inducible kinases), has been attributed to a wide diversity of essential biological processes in tumor, such as cell metabolism, polarity, differentiation, and proliferation..$^{29}$ It has been well identified that loss of LKB1 is related to poor prognosis in different types of cancer. ${ }^{30}$ Downregulation of LKB1 in neuroendocrine tumors of the lung has been reported nearly a decade ago, ${ }^{27}$ but the role of LKB1 in neuroendocrine tumors remains unclear. To our knowledge, this is the first study to investigate the correlation between LKB1 expression and clinicopathological features and prognosis in pNETs. We discovered a possible prognostic value for LKB1 expression in human pNETs.

The relationship between LKB1 expression levels and certain clinicopathological parameters was evaluated. Previous research revealed that LKB1 acts as a critical barrier to pulmonary tumorigenesis through inhibiting cell differentiation and epithelial-mesenchymal transition. ${ }^{31}$ LKB1 has also been implicated in regulating the differentiation of a number of other cell types, such as colonic epithelial cancer cell, pancreatic endocrine cell, endothelial progenitor cell, and neuronal cells. ${ }^{32-36}$ AMPK/mTOR pathway, a key downstream signal pathway of LKB1, was implicated as the most important mediator of LKB1-induced regulation of cell differentiation. ${ }^{37}$ More importantly, the role of mTOR 
Table I Correlation between LKBI expression and clinicopathological features in patients with pNET

\begin{tabular}{|c|c|c|c|c|}
\hline \multirow[t]{2}{*}{ Variables } & \multirow{2}{*}{$\begin{array}{l}\text { Patients } \\
(n=7 I)\end{array}$} & \multicolumn{2}{|c|}{ LKBI expression levels } & \multirow[t]{2}{*}{$P$-value ${ }^{b}$} \\
\hline & & $\begin{array}{l}\text { High } \\
(n=33)\end{array}$ & $\begin{array}{l}\text { Low or negative } \\
(n=38)\end{array}$ & \\
\hline Age, years (mean $\pm S D$ ) & $45.2 \pm 17.5$ & $40.3 \pm 15.1$ & $49.11 \pm 17.6$ & 0.042 \\
\hline Gender & & & & 0.088 \\
\hline Female & 29 & 17 & 12 & \\
\hline Male & 42 & 16 & 26 & \\
\hline Tumor size, $\mathrm{cm}($ mean $\pm \mathrm{SD})$ & $3.3 \pm 2.1$ & $2.70 \pm 1.73$ & $3.75 \pm 2.33$ & 0.307 \\
\hline Median (upper and lower quartiles) & & $2(0.4-7)$ & $3.45(0.8-9)$ & \\
\hline Function & & & & 0.085 \\
\hline Functional & 31 & 18 & 13 & \\
\hline Non-functional & 40 & 15 & 25 & \\
\hline Location & & & & 0.925 \\
\hline Head/uncinate & 34 & 16 & 18 & \\
\hline Body and/or tail & 37 & 17 & 20 & \\
\hline Margin status & & & & 0.348 \\
\hline RO & 70 & 33 & 37 & \\
\hline RI & 1 & 0 & I & \\
\hline Differentiation & & & & 0.072 \\
\hline Well/moderate & 64 & 32 & 32 & \\
\hline Poor & 7 & 1 & 6 & \\
\hline $\mathrm{Ki}-67$ index, \% & & & & 0.004 \\
\hline$\leq 2$ & 39 & 25 & 14 & \\
\hline $3-20$ & 26 & 7 & 19 & \\
\hline$>20$ & 6 & I & 5 & \\
\hline Mitotic count, per $10 \mathrm{HPF}$ & & & & 0.001 \\
\hline$<2$ & 38 & 28 & 10 & \\
\hline $2-20$ & 25 & 4 & 21 & \\
\hline$>20$ & 8 & I & 7 & \\
\hline G grade ${ }^{a}$ & & & & 0.001 \\
\hline GI & 31 & 24 & 7 & \\
\hline G2 & 30 & 7 & 23 & \\
\hline G3 & 10 & 2 & 8 & \\
\hline
\end{tabular}

Note: ${ }^{a}$ The histologic grade was classified according to WHO 2010 classification system; ${ }^{b} t$-test for continuous variables and chi-square test for categorical variables. Abbreviations: LKBI, liver kinase BI; pNET, pancreatic neuroendocrine tumor; HPF, high power field; WHO, World Health Organization.

pathway in pNETs has been well established. ${ }^{38,39}$ A wholegenome analysis with large sample size showed that mTOR signaling is one of the most common pathways displayed in somatic mutations. ${ }^{39}$ Additionally, several key negative regulators of the mTOR pathway have been identified to be downregulated in most of the primary pNETs, such as TSC2 and PTEN. ${ }^{40}$ In a previous study, marked reduction of LKB1 expression in high-grade neuroendocrine tumors of lung was observed. ${ }^{27}$ Our study revealed a similar result that reduced LKB1 expression in pNET was correlated with increased Ki-67 index, mitotic count, and advanced histologic grade.

The relationship between LKB1 expression levels and prognosis in pNET patients was also evaluated. Our study revealed that patients with a low level of LKB1 expression had significantly decreased OS and DFS. Additionally, our study revealed a similar phenomenon in the subgroup of patients with NF-pNET, in whom the recurrence is usually insidious due to the absence of endocrine symptoms. Our findings provided a potential additional marker that could help to guide the follow-up strategy. For example, among the pNET patients exposed to the same clinicopathological risk factors, such as histologic grade and tumor size, the cases with low LKB1 expression should be evaluated more carefully and frequently.

\section{Limitations}

There are some limitations in this study. First, our cohort included patients treated at our institution during an extended period of more than 16 years. The follow-up program was not standardized for every patient during the long time. For example, a less strict follow-up strategy may bias the time to detect recurrence. Second, due to the low incidence of pNETs, it is hard to obtain a cohort of large size in a single institution. Therefore, it is difficult for the sample size of this 
A

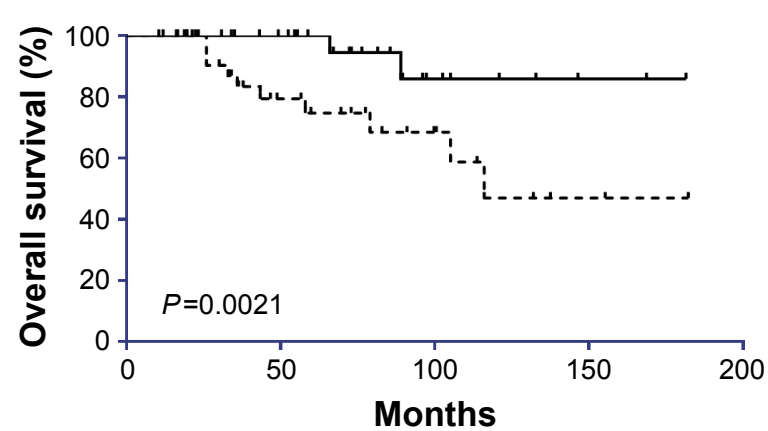

C

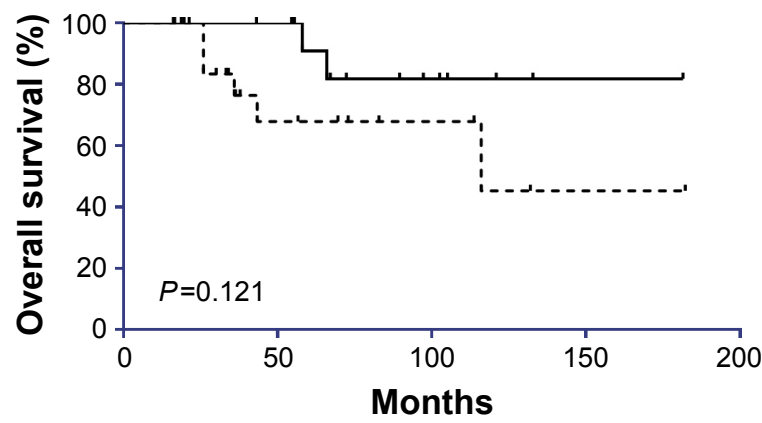

B

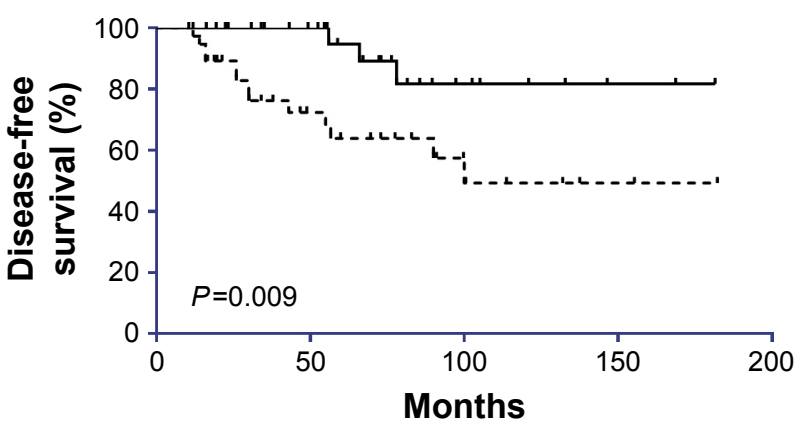

D

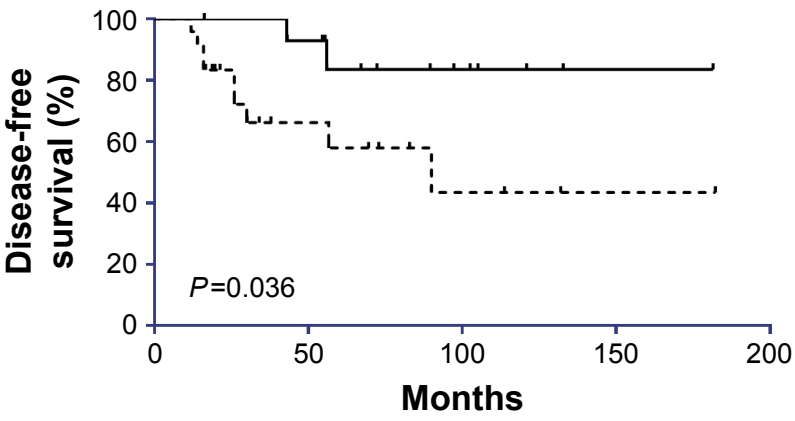

- High expression - - L. Low expression or negative expression

Figure 3 Correlation between LKBI and survival outcomes tested by Kaplan-Meier analysis and log-rank test.

Notes: (A) Patients with low/negative expression of LKBI had a significantly decreased OS than those with high expression (log-rank test, $P=0.002 \mathrm{I}$ ). (B) Patients with low/negative expression of LKBI had a significantly decreased DFS than those with high expression (log-rank test, $P=0.009$ ). (C) In patients with non-functional pancreatic neuroendocrine tumors (NF-pNETs), decreased LKBI expression was associated with poor OS (log-rank test, $P=0.121$ ). (D) In patients with NF-pNETs, decreased LKBI expression was associated with significantly shorter DFS (log-rank test, $P=0.036$ ).

Abbreviations: LKBI, liver kinase BI; OS, overall survival; DFS, disease-free survival.

study to reach the standard recommendations for Cox regression analysis. The abovementioned limitations are difficult to overcome given the low incidence and long course of pNETs. Further prospective and multicenter trials are warranted to discover prognosis factors for PNETs and reveal underlying mechanisms involved in the development of this tumor.

\section{Acknowledgment}

This study was supported by the grants of People's Livelihood Science and Technology Project, Special Project on the Integration of Industry, Education and Research of Guangzhou City (2014Y200509).

\section{Disclosure}

The authors report no conflicts of interest in this work.

\section{References}

1. Young K, Iyer R, Morganstein D, Chau I, Cunningham D, Starling N. Pancreatic neuroendocrine tumors: a review. Future Oncol. 2015;11(5): 853-864.

2. Simard EP, Ward EM, Siegel R, Jemal A. Cancers with increasing incidence trends in the United States: 1999 through 2008. CA Cancer J Clin. 2012;62(2):118-128.
3. Minter RM, Simeone DM. Contemporary management of nonfunctioning pancreatic neuroendocrine tumors. J Gastrointest Surg. 2012;16(2): $435-446$.

4. Falconi M, Eriksson B, Kaltsas G, et al. ENETS Consensus guidelines update for the management of patients with functional pancreatic neuroendocrine tumors and non-functional pancreatic neuroendocrine tumors. Neuroendocrinol. 2016;103(2):153-171.

5. Chiruvella A, Kooby DA. Surgical management of pancreatic neuroendocrine tumors. Surg Oncol Clin N Am. 2016;25(2):401-421.

6. Solorzano CC, Lee JE, Pisters PW, et al. Nonfunctioning islet cell carcinoma of the pancreas: survival results in a contemporary series of 163 patients. Surgery. 2001;130(6):1078-1085.

7. Haynes AB, Deshpande V, Ingkakul T, et al. Implications of incidentally discovered, nonfunctioning pancreatic endocrine tumors: short-term and long-term patient outcomes. Arch Surg. 2011;146(5):534-538.

8. Yao JC, Hassan M, Phan A, et al. One hundred years after "carcinoid": epidemiology of and prognostic factors for neuroendocrine tumors in 35,825 cases in the United States. J Clin Oncol. 2008;26(18): 3063-3072.

9. Wei IH, Harmon CM, Arcerito M, Cheng DF, Minter RM, Simeone DM. Tumor-associated macrophages are a useful biomarker to predict recurrence after surgical resection of nonfunctional pancreatic neuroendocrine tumors. Ann Surg. 2014;260(6):1088-1094.

10. Fitzgerald TL, Mosquera C, Vora HS, Vohra NA, Zervos EE. Indications for surgical resection in low-grade pancreatic neuroendocrine tumors. Am Surg. 2016;82(8):737-742.

11. Hemminki A, Markie D, Tomlinson I, et al. A serine/threonine kinase gene defective in Peutz-Jeghers syndrome. Nature. 1998;391(6663): 184-187. 
12. Jenne DE, Reimann H, Nezu J, et al. Peutz-Jeghers syndrome is caused by mutations in a novel serine threonine kinase. Nat Genet. 1998; 18(1):38-43.

13. Hardie DG. New roles for the LKB1-->AMPK pathway. Curr Opin Cell Biol. 2005;17(2):167-173.

14. Mirouse V, Billaud M. The LKB1/AMPK polarity pathway. FEBS Lett. 2011;585(7):981-985.

15. Nakano A, Takashima S. LKB1 and AMP-activated protein kinase: regulators of cell polarity. Genes Cells. 2012;17(9):737-747.

16. Udd L, Makela TP. LKB1 signaling in advancing cell differentiation. Fam Cancer. 2011;10(3):425-435.

17. Williams T, Brenman JE. LKB1 and AMPK in cell polarity and division. Trends Cell Biol. 2008;18(4):193-198.

18. Jansen M, Ten Klooster JP, Offerhaus GJ, Clevers H. LKB1 and AMPK family signaling: the intimate link between cell polarity and energy metabolism. Physiol Rev. 2009;89(3):777-798.

19. Yang JY, Jiang SH, Liu DJ, et al. Decreased LKB1 predicts poor prognosis in pancreatic ductal adenocarcinoma. Sci Rep. 2015;5:10575.

20. Linher-Melville K, Zantinge S, Singh G. Liver kinase B1 expression (LKB1) is repressed by estrogen receptor alpha (ERalpha) in MCF-7 human breast cancer cells. Biochem Biophys Res Commun. 2012;417(3): 1063-1068.

21. Mack HI, Munger K. The LKB1 tumor suppressor differentially affects anchorage independent growth of HPV positive cervical cancer cell lines. Virology. 2013;446(1-2):9-16.

22. Tigli H, Seven D, Tunc M, et al. LKB1 mutations and their correlation with LKB1 and Rheb expression in bladder cancer. Mol Carcinog. 2013; 52(8):660-665.

23. Gross RW, Patti GJ, Milbrandt J, et al. Loss of LKB1 and PTEN tumor suppressor genes in the ovarian surface epithelium induces papillary serous ovarian cancer. Nat Neurosci. 2014;35(3):546-553.

24. He TY, Tsai LH, Huang CC, Chou MC, Lee H. LKB1 loss at transcriptional level promotes tumor malignancy and poor patient outcomes in colorectal cancer. Ann Surg Oncol. 2014;21 (Suppl 4): S703-S710.

25. Li F, Han X, Li F, et al. LKB1 Inactivation Elicits a redox imbalance to modulate non-small cell lung cancer plasticity and therapeutic response. Cancer Cell. 2015;27(5):698-711.
26. Huang YH, Chen ZK, Huang KT, et al. Decreased expression of LKB1 correlates with poor prognosis in hepatocellular carcinoma patients undergoing hepatectomy. APJCP. 2013;14(3):1985-1988.

27. Amin RM, Hiroshima K, Iyoda A, et al. LKB1 protein expression in neuroendocrine tumors of the lung. Pathol Int. 2008;58(2):84-88.

28. Sanchez-Cespedes M. A role for LKB1 gene in human cancer beyond the Peutz-Jeghers syndrome. Oncogene. 2007;26(57):7825-7832.

29. Korsse SE, Peppelenbosch MP, van Veelen W. Targeting LKB1 signaling in cancer. Biochimi Biophys Acta. 2013;1835(2):194-210.

30. Gan RY, Li HB. Recent progress on liver kinase B1 (LKB1): expression, regulation, downstream signaling and cancer suppressive function. Int J Mol Sci. 2014;15(9):16698-16718.

31. Ji H, Ramsey MR, Hayes DN, et al. LKB1 modulates lung cancer differentiation and metastasis. Nature. 2007;448(7155):807-810.

32. Kan JY, Yen MC, Wang JY, et al. Nesfatin-1/Nucleobindin-2 enhances cell migration, invasion, and epithelial-mesenchymal transition via LKB1/ AMPK/TORC1/ZEB1 pathways in colon cancer. Oncotarget. 2016;7(21): 31336-31349.

33. de Wilde RF, Ottenhof NA, Jansen M, et al. Analysis of LKB1 mutations and other molecular alterations in pancreatic acinar cell carcinoma. Mod Pathol. 2011;24(9):1229-1236.

34. Kone M, Pullen TJ, Sun G, et al. LKB1 and AMPK differentially regulate pancreatic beta-cell identity. FASEB J. 2014;28(11):4972-4985.

35. Nakada D, Saunders TL, Morrison SJ. Lkb1 regulates cell cycle and energy metabolism in haematopoietic stem cells. Nature. 2010;468(7324): 653-658.

36. Shelly M, Poo MM. Role of LKB1-SAD/MARK pathway in neuronal polarization. Dev Neurobiol. 2011;71(6):508-527.

37. Shaw RJ, Bardeesy N, Manning BD, et al. The LKB1 tumor suppressor negatively regulates mTOR signaling. Cancer Cell. 2004;6(1):91-99.

38. Vandamme T, Peeters M, Dogan F, et al. Whole-exome characterization of pancreatic neuroendocrine tumor cell lines BON-1 and QGP-1. J Mol Endocrinol. 2015;54(2):137-147.

39. Scarpa A, Chang DK, Nones K, et al. Whole-genome landscape of pancreatic neuroendocrine tumours. Nature. 2017;543(7643):65-71.

40. Missiaglia E, Dalai I, Barbi S, et al. Pancreatic endocrine tumors: expression profiling evidences a role for AKT-mTOR pathway. J Clin Oncol. 2010;28(2):245-255.
OncoTargets and Therapy

\section{Publish your work in this journal}

OncoTargets and Therapy is an international, peer-reviewed, open access journal focusing on the pathological basis of all cancers, potential targets for therapy and treatment protocols employed to improve the management of cancer patients. The journal also focuses on the impact of management programs and new therapeutic agents and protocols on

\section{Dovepress}

patient perspectives such as quality of life, adherence and satisfaction. The manuscript management system is completely online and includes a very quick and fair peer-review system, which is all easy to use. Visit http://www.dovepress.com/testimonials.php to read real quotes from published authors. 